\title{
Depressione e patologia renale: rassegna della letteratura e prospettive di intervento
}

\author{
F. Logias ${ }^{1}$, M.J. Sequenza ${ }^{2}$, A. Granata ${ }^{3}$, D. Soru ${ }^{4}$ \\ ${ }^{1}$ U.O.C. Nefrologia e Dialisi, Ospedale "S. Francesco", Nuoro \\ ${ }^{2}$ Farmacia di Atzara, Atzara (NU) \\ ${ }^{3}$ U.O.C. Nefrologia e Dialisi, Ospedale "San Giovanni di Dio”, Agrigento \\ ${ }^{4}$ Dipartimento di Pedagogia, Psicologia e Filosofia, Università degli Studi di Cagliari, Cagliari
}

\begin{abstract}
DEPRESSION AND RENAL PATHOLOGY: LITERATURE REVIEW AND PERSPECTIVES FOR INTERVENTION
Aвstract. Depression in patients affected by renal disease is a relevant problem because it is related to a increase of death and hospitalization rate and to a reduction of compliance. The pharmacological treatments result to be the best therapy of choice, however there is a greater difficulty in treating depressive symptoms that occur in comorbidity with renal disease, than when depression is the only disease. Various studies show that this problem can be addressed through an integrated pharmacological and psychotherapeutic intervention, which presents an additive effect in the treatment of this pathology. In relation to renal disease various studies suggest the effectiveness of psychotherapeutic intervention in the treatment of depressive symptoms, but more research is needed to compare the effectiveness of the different psychotherapeutic approaches.
\end{abstract}

Key Words. Depression in renal disease, Compliance, Antidepressant drugs, Psychotherapy and depression

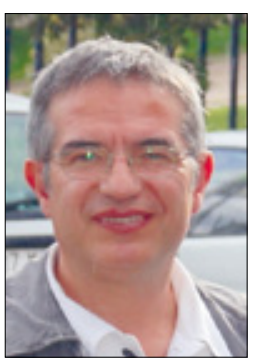

Franco Logias

\section{Depressione e decorso della patologia renale}

Diversi studi hanno indagato la relazione tra disagio psichico e patologia renale, in particolare rispetto alla presenza di sintomi depressivi. La depressione è inserita, nella classificazione del DSM-IV-TR (1), all'interno della classe dei disturbi dell'umore e può essere distinta in categorie quali ad esempio il disturbo depressivo maggiore e il disturbo distimico, oppure può essere classificata in un continuum che va da sintomi tra loro isolati fino al disturbo bipolare. Il disturbo depressivo maggiore è caratterizzato dalla presenza, per almeno due settimane, di uno o più episodi depressivi maggiori, in cui sono presenti almeno 5 dei seguenti sintomi:

- umore depresso per la maggior parte del giorno;

- sensibile diminuzione per tutte o per la maggior parte delle attività;

- significativa perdita o aumento di peso senza che si segua una particolare dieta;
- insonnia o ipersonnia quasi ogni giorno;

- osservabile agitazione o rallentamento psicomotorio;

- mancanza di energia o faticabilità per quasi tutto il giorno;

- sentimenti di auto-svalutazione o colpa eccessiva quasi ogni giorno;

- ridotta capacità di pensare o concentrarsi quasi ogni giorno;

- pensieri ricorrenti di morte o suicidio, con l'eventuale presenza di atti suicidari.

Il disturbo distimico è caratterizzato dalla presenza, per almeno due anni, di un umore depresso pressoché quotidiano che è associato a sintomi depressivi che non soddisfano i criteri per il disturbo depressivo maggiore. La presenza e la riduzione dei sintomi depressivi può essere valutata attraverso un'intervista strutturata che segue i criteri del DSM-IV-TR. Vengono tuttavia maggiormente utilizzati altri strumenti, che consentono di misurare i sintomi depressivi in maniera più rapida, quali, ad esempio, $\mathrm{i}$ punteggi ottenuti in diverse scale auto-somministrate, tra cui spiccano la Back Depression Inventory (nella versione II) e la Hamilton Depression Rating Scale. 
La presenza di depressione è stata infatti descritta come il più importante problema psichiatrico nella patologia renale, in particolare nella fase terminale (2). Essa è correlata all'aumento di rischio di decesso e ospedalizzazione (3), anche a seguito di condotte suicidarie $(4,5)$, sia per i pazienti in trattamento emodialitico che per quelli in dialisi peritoneale (6).

Uno studio (3) evidenzia, per l'insufficienza renale terminale, una relazione tra depressione e tasso di decesso o ospedalizzazione, anche dopo aver controllato per "età", "genere", "tempo di dialisi" e comorbidità. Tale associazione, in relazione alla depressione maggiore, risulta anche per pazienti che non sono in trattamento dialitico e che presentano insufficienza renale a partire dallo stadio 2 (7). Per quanto riguarda i comportamenti suicidari, seppur se ne possa evidenziare una diminuzione in pazienti con insufficienza renale terminale (4), il suicidio in tali pazienti risulta più alto rispetto alla popolazione generale (8) ed è riscontrabile un rischio rappresentato dal persistere di pensieri suicidari (9), che cresce con la gravità dei sintomi depressivi (10). La presenza di episodi depressivi maggiori, assieme all'agorafobia, rappresenta per i pazienti in emodialisi un fattore di rischio per i comportamenti suicidari, annullando anche l'effetto della presenza di una fede religiosa che è un fattore di protezione (11).

Diversi studi evidenziano inoltre una relazione tra depressione e compliance terapeutica. Uno studio metaanalitico (12) mostra il collegamento tra depressione e non aderenza in pazienti affetti da patologie croniche: coloro che soffrono di depressione presentano, rispetto a coloro che non ne sono affetti, 1.76 volte l'odds ratio di non aderenza. Uno studio specifico sulla patologia renale (13) suggerisce che la depressione è il terzo fattore più importante (dopo "genere" e "modalità di trattamento", Dialisi vs Trapianto) che permette di predire la compliance. Un altro fattore infine che, assieme alla depressione, permette di predire la compliance, è rappresentato da una ridotta performance dei pazienti nei test di problem-solving quotidiano (14). Essa è inoltre uno dei possibili fattori associati alla non compliance rispetto alle prescrizioni alimentari (15) ed è collegata sia alla non aderenza che alla mortalità (16).

La depressione potrebbe essere legata alla malattia cronica, poiché le persone affette da patologie croniche presentano livelli di stress superiori alla media della popolazione (17), che possono sfociare anche in problemi di disagio psichico. Per ciò che riguarda la patologia renale si possono individuare diversi fattori di stress quali limitazioni dietetiche, limitazioni temporali imposte dalla dialisi, limitazioni funzionali, eventuale perdita del lavoro e del proprio ruolo nella società, eventuali cambiamenti dello stile di vita e della propria percezione di salute, ripensamento diverso della propria immagine corporea.

Se quindi la depressione è un importante fattore che ha un effetto sulla ospedalizzazione, sul decesso, sui comportamenti suicidari e sulla compliance in relazione alla patologia renale diventa importante riconoscerla precocemente in modo da avviare un trattamento che consenta di intervenire in maniera efficace.

\section{Prospettive di intervento farmacologico}

Sono state identificate varie tipologie di trattamenti per i sintomi depressivi. Quello elettivo rimane il trattamento farmacologico, attraverso antidepressivi triciclici (TCA), inibitori selettivi della ricaptazione della serotonina (SSRI), inibitori della ricaptazione della serotoninanorepinefrina (SNRI) e antidepressivi atipici.

In relazione agli effetti degli antidepressivi sono stati pubblicati vari studi molti dei quali, oltre a valutarne l'efficacia, approfondiscono la questione di quale farmaco presenti il migliore rapporto costi-benefici. Uno studio (18) analizza, in tale direzione, gli articoli pubblicati tra il 1999 e la fine del 2010 che si occupano di questa tematica. Sono stati considerati studi basati su database, trial controllati randomizzati e studi osservazionali che comportassero sia l'analisi comparata di diversi tipi di antidepressivi sia l'indicazione dei costi. In questo studio l'Escitalopram emerge come il farmaco che, tra gli SSRI e gli SNRI, mostra la maggiore persistenza al trattamento, il minore tasso di ospedalizzazione e il minore costo sanitario. Per quanto riguarda gli SNRI si riscontra che la Venlafaxina XR presenta la maggiore persistenza al farmaco rispetto agli altri SSRI (ad eccezione dell'Escitalopram) e un costo equiparabile sia a tali SSRI sia alla Venlafaxina. Per quanto riguarda la differenza tra gli SSRI e TCA si evidenzia che i costi dei TCA sono equiparabili a quelli degli SSRI, tuttavia alcune ricerche mostrano che l'utilizzo degli SSRI è associato a maggiore persistenza al trattamento e minori costi, mentre altri evidenziano che, in trial pragmatici, i TCA hanno minori costi degli SSRI e outcome simili o migliori di questi ultimi: tali studi suggeriscono quindi che questo aspetto meriti un ulteriore approfondimento in future ricerche.

Per quanto concerne l'effetto specifico dei farmaci antidepressivi su pazienti nefropatici (a partire dallo stadio 3 dell'insufficienza renale) una recente rassegna (19), seppur con dei limiti rappresentati dalla presenza di uno scarso numero di trial clinici randomizzati, suggerisce l'assenza di evidenze sufficienti che mostrino che gli antidepressivi siano migliori dei placebo nel trattare la depressione in pazienti con CKD 3-5, mentre alcuni studi osservativi e prospettici suggeriscono che il trattamento farmacologico migliora i 
sintomi depressivi dopo 8-12 settimane, riportando tuttavia una dimensione dell'effetto simile a quella del placebo. Ciò potrebbe essere dovuto al fatto che, quando la depressione è associata anche a problemi renali, essa è maggiormente resistente al trattamento rispetto a quando essa è l'unico disturbo presente (20), tuttavia una recente rassegna (21) evidenzia una riduzione della depressione in seguito a somministrazione di S-adenosil metionina e di Paroxetina.

In relazione al dosaggio si evidenzia che la clearance dei principali antidepressivi risulta alterata in pazienti con insufficienza renale cronica: è quindi importante una riduzione della dose sia per quanto riguarda i TCA, sia per gli SSRI che per gli SNRI, in particolare a partire dallo stadio 4 dell'insufficienza renale cronica. Per quanto concerne più dettagliatamente l'Escitolpram è importante tener conto che esso dovrebbe essere utilizzato con estrema cautela in pazienti con grave insufficienza renale, poiché può causare un prolungamento dell'intervallo QT e torsioni di punta (22).

\section{Prospettive di intervento psicoterapeuti- co e integrato}

La letteratura relativa all'intervento psicoterapeutico in relazione al trattamento della depressione presenta risultati eterogenei ma promettenti, soprattutto per quanto riguarda l'associazione tra psicoterapia e interventi farmacologici. Sono state riscontrate delle evidenze in relazione a differenti approcci psicoterapeutici, le cui principali caratteristiche sono riassunte nella Tabella I. In una ricerca (23) è stata analizzata, nell'arco di 12 settimane, l'efficacia di un trattamento psicoterapeutico di tipo cognitivo-comportamentale (CBASP) rispetto a un

\section{TABELLA I - CARATTERISTICHE DEI PRINCIPALI APPROCCI PSICOTERAPEUTICI QUI DISCUSSI}

\begin{tabular}{|c|c|}
\hline Approccio & Principali caratteristiche \\
\hline $\begin{array}{l}\text { Psicoterapia Psicodinamica Breve } \\
\text { (BPP) }\end{array}$ & $\begin{array}{l}\text { Ventaglio di proposte psicoterapeutiche che considerano i sintomi principalmente come soluzioni } \\
\text { a conflitti inconsci del paziente, associati per lo più alle relazioni con le persone per lui significative } \\
\text { interiorizzate nel corso della vita. Obiettivi principali dell'intervento sono: } \\
\text { 1) Analizzare l'organizzazione di personalità del paziente secondo un modello psicodinamico; } \\
\text { 2) Individuare uno o più problemi centrali su cui lavorare; } \\
\text { 3) Aiutare il paziente a far emergere i conflitti e i vissuti inconsci che influenzano il suo comportamento } \\
\text { (psicoterapia principalmente espressiva) oppure a sviluppare le risorse che già possiede per ristabilire } \\
\text { un equilibrio omeostatico interno e con l'ambiente (psicoterapia principalmente supportiva). }\end{array}$ \\
\hline $\begin{array}{l}\text { Psicoterapia Cognitivo Comportamentale } \\
\text { (CBT) }\end{array}$ & $\begin{array}{l}\text { Approccio psicoterapeutico che concettualizza i sintomi come risultante di pensieri e comportamenti } \\
\text { non adattivi. L'obiettivo della psicoterapia è analizzare i pensieri associati ai sintomi e alle situazioni } \\
\text { in cui emergono, per poi modificarli e ottenere un beneficio sia sui sintomi che sui comportamenti } \\
\text { associati. }\end{array}$ \\
\hline Psicoterapia Interpersonale (IPT) & $\begin{array}{l}\text { Psicoterapia breve di matrice sia psicodinamica che cognitivo-comportamentale, altamente strutturata } \\
\text { e sviluppata originariamente per il trattamento della depressione. La durata è limitata (da } 6 \text { a } 20 \\
\text { settimane) e il focus è su un numero ristretto di fattori, legati alle relazioni interpersonali, ritenuti } \\
\text { importanti nella genesi del malessere del paziente. L'obiettivo principale è la risoluzione dei sintomi } \\
\text { presentati. }\end{array}$ \\
\hline
\end{tabular}


trattamento psicoterapeutico supportivo breve (BSP), integrato o meno con un trattamento farmacologico. Il campione è costituito da 491 partecipanti che, nelle 12 settimane precedenti la psicoterapia, non hanno risposto o hanno risposto soltanto parzialmente al trattamento farmacologico. I risultati di questo trial randomizzato evidenziano che il trattamento psicoterapeutico non permette di osservare una differenza statisticamente significativa tra le tre condizioni (trattamento farmacologico, CBASP, BSP) in relazione alle remissioni, suggerendo quindi che la psicoterapia non produca dei miglioramenti rispetto al trattamento farmacologico.

Un'altra ricerca tuttavia (24) approfondisce, attraverso una meta-analisi su 16 trial randomizzati, gli effetti di 6 tipologie di trattamento psicoterapeutico su pazienti con depressione maggiore e distimia, in relazione alla terapia farmacologica. I risultati mostrano che la psicoterapia ha un effetto significativo (seppur la dimensione dell'effetto sia minore di quella relativa al trattamento farmacologico) rispetto ai gruppi di controllo. Il trattamento combinato (farmacologico e psicoterapeutico) risulta migliore sia del trattamento farmacologico (per pazienti con depressione maggiore ma non con distimia), sia del solo trattamento psicoterapeutico. È stata inoltre analizzata, attraverso una meta-analisi (25), l'efficacia di un intervento psicoterapeutico sulla depressione in pazienti ricoverati, sottoposti quindi alle classiche terapie farmacologiche e occupazionali, riscontrando ancora una volta la presenza di un effetto aggiuntivo piccolo ma statisticamente significativo rispetto alle altre terapie.

Diverse ricerche hanno indagato l'effetto di singoli approcci psicoterapeutici. Per quanto concerne la psicoterapia psicodinamica breve uno studio meta-analitico (26) evidenzia che essa ha un effetto statisticamente significativo sul trattamento della depressione rispetto ai gruppi di controllo. Tale effetto è mantenuto dopo 3 mesi, 6 mesi e un anno di follow-up. Tale tipologia di psicoterapia si dimostra superiore quando è individuale piuttosto che di gruppo, probabilmente perché la psicoterapia individuale è maggiormente adattata ai bisogni di ogni singolo paziente. In relazione ad altri tipi di psicoterapia lo studio rileva una lieve superiorità di queste ultime ma tale effetto è statisticamente non significativo dopo un anno di follow-up.

Una meta-analisi (27) sull'efficacia di una specifica psicoterapia psicodinamica breve, la terapia interpersonale (IPT), evidenzia una efficacia della IPT rispetto al placebo e ai gruppi di controllo, una differenza non statisticamente significativa tra l'IPT e altri tipi di psicoterapie, una maggiore efficacia della terapia farmacologica (in particolare degli SSRI) rispetto all'IPT, una differenza statisticamente significativa tra l'uso combinato di IPT e terapia farmacologica, rispetto alla sola terapia farmacologica, soltanto quando la variabile dipendente era operazionalizzata analizzando i punteggi ottenuti nella scala HDRS.

Per quanto concerne l'efficacia della psicoterapia per pazienti con insufficienza renale uno studio su un campione randomizzato, in pazienti che hanno ricevuto un trapianto di rene da donatore cadavere (28), evidenzia una maggiore riduzione dei sintomi depressivi in pazienti trattati con una psicoterapia sistemica individuale sia rispetto a quelli trattati con una psicoterapia sistemica di gruppo che al gruppo di controllo. Un altro studio su pazienti in emodialisi, trattati con una approccio di gruppo di tipo cognitivo comportamentale (29) evidenzia, rispetto al gruppo di controllo, una riduzione dei sintomi depressivi dopo 3 mesi di trattamento.

Globalmente questi studi evidenziano, da un punto di vista generale, l'efficacia di un trattamento integrato (farmacologico e psicoterapeutico) in relazione alla cura della depressione, mettendo in luce l'importanza di un intervento psicoterapeutico anche in pazienti con patologia renale. Si rendono necessari ulteriori studi per valutare quale tra i vari trattamenti psicoterapeutici esistenti sia il più efficace per il trattamento della depressione in pazienti con insufficienza renale.

\section{Conclusioni}

L'analisi della letteratura mostra l'importanza di rilevare e trattare la presenza di sintomi depressivi in relazione al decorso della patologia renale, sia per quanto riguarda il tasso di decesso e ospedalizzazione che per ciò che concerne la compliance farmacologica e alimentare. La depressione, quando si presenta in comorbidità con la patologia renale, risulta più difficile da trattare rispetto a quando essa è l'unico disturbo presente. Il trattamento farmacologico risulta essere la terapia elettiva, tuttavia si evidenzia che l'associazione ai farmaci di un intervento psicoterapeutico permette di alleviare i sintomi depressivi nella popolazione generale. Per quanto riguarda la patologia renale diversi studi suggeriscono l'efficacia di un trattamento psicoterapeutico nel trattamento dei sintomi depressivi. L'esiguo numero di ricerche effettuate, nonché la valutazione della presenza di sintomi depressivi attraverso scale tra loro differenti e non sempre comparabili, evidenziano l'importanza di ulteriori studi che confrontino i differenti approcci psicoterapeutici nella riduzione dei sintomi depressivi. Altresì sembra importante definire una scala di misurazione condivisa della depressione che permetta una valutazione comparata degli approcci farmacologici e di quelli psicoterapeutici. 


\section{Riassunto}

La depressione nei pazienti affetti da patologia renale è un problema rilevante poiché essa è associata a un aumento del tasso di decesso e ospedalizzazione e a una riduzione della compliance. Il trattamento farmacologico si presenta come la terapia elettiva, tuttavia si riscontra una maggiore difficoltà nel trattare $\mathrm{i}$ sintomi depressivi che si presentano in comorbidità con la patologia renale rispetto a quando la depressione è l'unica patologia. Diverse ricerche mostrano che questo problema può essere affrontato attraverso un intervento integrato farmacologico e psicoterapeutico, il quale ha un effetto additivo nel trattamento di questa patologia. Per quanto riguarda la patologia renale diversi studi suggeriscono l'efficacia di un intervento psicoterapeutico nel trattamento dei sintomi depressivi, tuttavia sono necessarie ulteriori ricerche che confrontino l'efficacia dei diversi approcci psicoterapeutici.

Parole Chiave. Depressione nella patologia renale, Compliance, Farmaci antidepressivi, Psicoterapia e depressione

Indirizzo degli Autori:

Dr. Franco Logias

Via Mannironi

08100 Nuoro

logias@tiscali.it

\section{Bibliografia}

1. American Psychiatric Association. Diagnostic and Statistical Manual of Mental Disorders DSM-IV-TR Fourth Edition (Text Revision). Arlington, VA, American Psychiatric Association Pub, 2000.

2. Kimmel PL, Weihs K, Peterson RA. Survival in Hemodialysis Patients: The Role of Depression. JASN 1993; 4(1): 12-27.

3. Hedayati SS, Bosworth HB, Briley LP, et al. Death or hospitalization of patients on chronic hemodialysis is associated with a physician-based diagnosis of depression. Kidney Int 2008; 74: 930-36.

4. Kimmel PL. Depression in patients with chronic renal disease: what we know and what we need to know. J Psychosom Res 2002; 53(4): 951-6.

5. Kimmel PL, Peterson R. Depression in end-stage re- nal disease patients treated with hemodialysis: Tools, correlates, outcomes and needs. Semin Dialysis 2005; 18: 91-7.

6. Einwohner R, Bernadini J, Fried L, Piraino B. The effect of depressive symptoms on survival in peritoneal dialysis patients. Perit Dial Int 2004; 24 (3): 256-63.

7. Hedayati SS, Minhajuddin AT, Afshar M, Toto RD, Trivedi MH, Rush AJ. Association Between Major Depressive Episodes in Patients With Chronic Kidney Disease and Initiatio of Dialysis, Hospitalization, or Death. JAMA 2010; 303(19): 1946-53.

8. Kurella M, Kimmel PL, Young BS, Chertow GM. Suicide in the United States end-stage renal disease program. J Am Soc Nephrol 2005; 16(3): 774-81.

9. Soykan A, Arapaslan B, Kumbasar H. Suicidal Behavior, Satisfaction With Life, and Perceived Social Support in End-Stage Renal Disease. Transplant Proc 2003; 35: 1290-1.

10. Keskin G, Engin E. The evaluation of depression, suicidal ideation and coping strategies in haemodialysis patients with renal failure. J Clin Nurs 2011; 20 (1920): 2721-32.

11. Martiny C, De Oliveira e Silva AC, Simões Neto JP, Nardi AE. Factors associated with risk of suicide in patients with hemodialysis. Compr Psychiatry 2011; 52(5): 465-8.

12. Grenard JL, Munjas BA, Adams JL, et al. Depression and Medication Adherence in the Treatment of Chronic Diseases in the United States: A Meta-Analysis. J Gen Intern Med 2011; 26(10): 1175-82.

13. Cukor D, Rosenthal DS, Jindal RM, Brown CD, Kimmel PL. Depression is an important contributor to low medication adherence in hemodialyzed patients and transplants recipents. Kidney Int 2009; 75: 1223-29.

14. Gelb, SR, Shapiro RJ, Thornton WJL. Predicting Medication Adherence and Employment Status Following Kidney Transplant: The Relative Utility of Traditional and Everyday Cognitive Approaches. Neuropsychology 2010; 24(4): 514-26.

15. Khalil AA, Frazier SK, Lennie TA, Sawaya BP. Depressive symptoms and dietary adherence in patients with end-stage renal disease. J Ren Care 2011; 37(1): 30-9.

16. Rosenthal Asher D, Ver Halen N, Cukor D. Depression and nonadherence predict mortality in hemodialysis treated end-stage renal disease patients. Hemodial Int 2012; 16: 387-93.

17. Verhaak PFM, Heijmans MJWM, Peters L, Rijken M. Chronic disease and mental disorder. Soc Sci Med 2005; 60: 789-97.

18. Pan YJ, Knapp M, McCrone P. Cost-effectiveness comparisons between antidepressant treatments in depression: Evidence from database analyses and 
prospective studies. J Affect Disord 2012; 139(2): 11325.

19. Nagler EV, Webster AC, Vanholder R, et al. Antidepressants for depression in stage 3-5 chronic kidney disease: a systematic review of pharmacokinetics, efficacy and safety with recommendations by European Renal Best Practice (ERBP). Nephrol Dial Transplant 2012; 27 (10): 3736-45.

20. Fallon M. Depression in end-stage renal disease. J Psychosoc Nurs Ment Health Serv 2011; 49(8): 30-34.

21. Vecchio M, Palmer SC, Tonelli M, Johnson DW, et al. Depression and sexual dysfunction in chronic kidney disease: a narrative review of the evidence in areas of significant unmet need. Nephrol Dial Transplant 2012; 27: 3420-28.

22. Hedayati SS, Yalamanchili V, Finkelstein FO. A practical approach to the treatment of depression in patients with chronic kidney disease and end-stage renal disease. Kidney Int 2012; 81(3): 247-55.

23. Kocsis JH, Gelenberg AJ, Rothbaum BO, et al. Cognitive Behavioral Analysis System of Psychotherapy and Brief Supportive Psychotherapy for Augmentation of Antidepressant Nonresponse in Chronic Depression. Arch Gen Psychiatry 2009; 66(11): 1178-88.
24. Cuijpers P, van Straten A, Schuurmans J, van Oppen P, Hollon SD, Andersson G. Psychotherapy for chronic major depression and dysthymia: a meta-analysis. Clin Psychol Rev 2010; 30(1): 51-62.

25. Cuijpers P, Clignet F, van Meijel B, van Straten A, Li J, Andersson G. Psychological treatment of depression in inpatients: A systematic review and meta-analysis. Clin Psychol Rev 2011; 31(3): 353-60.

26. Driessen E, Cuijpers P, de Maat SC, Abbass AA, de Jonghe F, Dekker JJ. The efficacy of short-term psychodynamic psychotherapy for depression: A metaanalysis. Clin Psychol Rev 2010; 30(1): 25-36.

27. Cuijpers P, Geraedts AS, van Oppen P, Andersson G, Markowitz JC, van Straten A. Interpersonal psychotherapy for depression: a meta-analysis. Am J Psychiatry 2011;168(6): 581-92.

28. Baines LS, Joseph JT, Jindal RM. Prospective randomized study of individual and group psychotherapy versus controls in recipients of renal transplants. Kidney Int 2004; 65:1937-42.

29. Duarte PS, Miyazaki MC, Luís Blay S, Sesso R. Cognitive-behavioral group therapy is an effective treatment for major depression in hemodialysis patients. Kidney Int 2009; 76: 414-21. 